\title{
Study on automatic extraction of bottlenose dolphin whistles from the background of
}

\author{
ocean noise \\ Xue Rui-chao ${ }^{1}$, Niu Fu-qiang ${ }^{1}$, Yang Yan-ming ${ }^{1}$, Huang Yue-kun ${ }^{1}$, Liu Wei ${ }^{2}$ \\ 1.Third Institute of Oceanography, State Oceanic Administration, Xiamen 361005, Fujian, China. \\ 2.The Reserve Management Office of Chinese White Dolphins and Amphioxus in Xiamen, Xiamen 361005, Fujian,
}

China.

\begin{abstract}
Bottlenose dolphins (Tursiops aduncus) use the frequency contour of whistles to identify each other and communicate information. In order to research the characteristics of whistles, as much as possible sound data needed. However, there is very difficult to extraction whistles from strong background of ocean noise. Manual recognition and extraction whistles are not only time-consuming, but also lost effective signals with low signal-to-noise ratio. Methods of the fully automatic detection and extraction of whistles are proposed in this paper. The entire process for noise cancellation to a spectrogram of sound data consists of eight steps. Experimental results show that the total extraction accuracy of the method is $92.5 \%$, and the effective extraction accuracy is $80.5 \%$. This method can meet the requirement of most data processing, and provide a certain technical support for acoustic research of marine mammals.
\end{abstract}

Keywords: Dolphin Whistles, Signal Extraction, Noise Cancellation, Acoustic Signal Processing

\section{Introduction}

Sounds produced by the biological sonar of dolphins have functions related to navigation and communication. They can also understand the shape and nature of the object[1], even under difficult conditions such as high noise and cluttered environments[2]. The operating distance and positioning accuracy of dolphins' sonar are better performance than the artificial ones. However, in order to investigate the echolocation system of these mammals, acoustical experiments are valuable and were conducted to study sound characteristics of dolphins.

The earliest acoustic studies of dolphins successfully recorded the sound signal of dolphins [1], dividing the dolphin signals into three categories: localization sound (click), communication sound (whistle) and emergency sound (burst pulse) [2] [3]. On the other hand, the sounds also mainly divide in two broad categories: pulsed and tonal sounds [4]. Whistles are tonal sounds, and clicks are pulsed sounds. Most dolphin species produce clicks vocalizations in addition to whistles. In this paper, we concern about the whistles which play an important underwater communication function role. The frequency range of whistles can vary from a few hundred hertz up to tens of kilohertz. But there is very difficult to extraction whistles from strong background of ocean noise. Manual recognition and extraction are not only time-consuming, but also lost effective signals with low signal-to-noise ratio. So in this paper we developed an automatic extraction algorithm which including eight steps. After processing, we can get clear images and information of signals. The brief steps are as follows: firstly, a series of noise cancellation techniques are applied to a spectrogram; secondly, recognition the whistles and extract the outline; finally, record the frequency range and the presentation time.

Despite the fact that a few studies had described acoustic parameter characteristics of dolphin whistles, the variability of the whistles are still unknown. We conducted a study on the automatic extraction of bottlenose dolphin whistles, which can lay a foundation for data accumulation.

\section{Methods}

The dolphin whistles automatic extraction algorithm described in this paper, is based on MATLAB (2013a version). We have drawn on the experience of some of our predecessors[5][6][7]. The extraction and identification algorithm includes eight steps. They are time-frequency processing, click removal, median filtering, background noise reduction, gaussian smoothing, threshold control, binaryzation method and graph detection of contours. The sample data we use in this paper is an audio file in wav format, with a sampling rate of $80 \mathrm{kHz}$ and duration of $2.2 \mathrm{~s}$. 
It contains four whistle signals. The results are shown in the following figures. Each step is described in detail as below.

\section{A. Data collection}

The data used in the paper was mainly downloaded from the internet and measured in the Dolphin Protection Base on the Fire Island of Xiamen. The download data includes 12 species of dolphins, mainly including bottlenose dolphins, Atlantic spotted dolphins, spotted dolphins, spiral spotted dolphins and the others. The experimental subject on the island are two bottlenose dolphins (Tursiops aduncus), one is 19 years old, another is 23 years old.
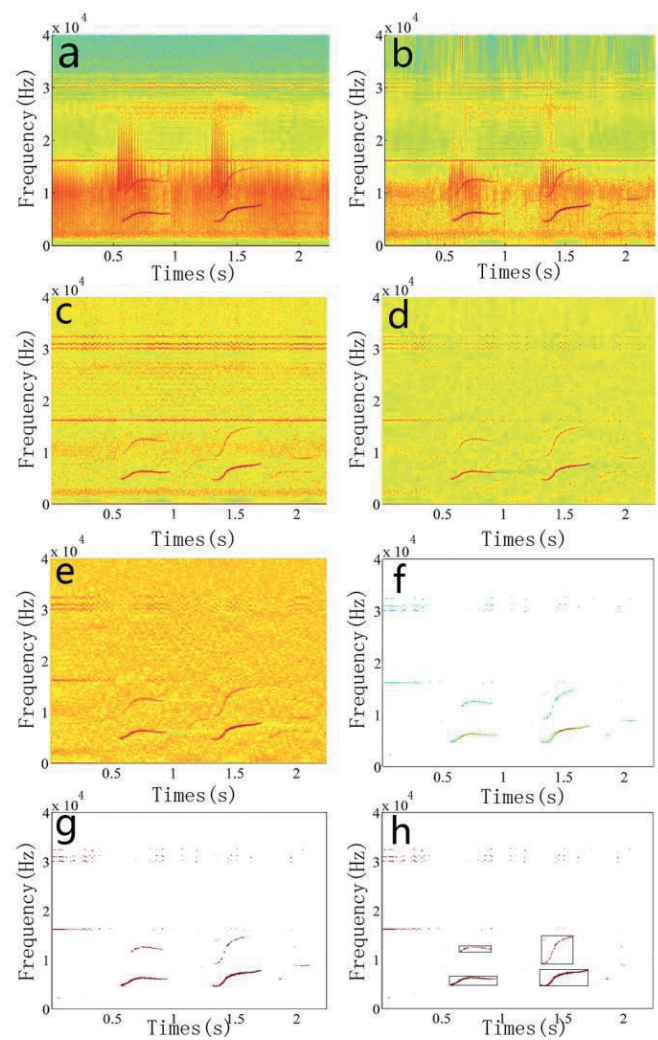

FIG.1. Spectrogram of a typical dolphin whistle showing the effect of the different processing steps of whistle contour extraction.

\section{B. Whistle extraction}

a) Time-frequency processing

To build the T-F spectrogram image, we use the FFT algorithm (the hamming window, length 1024).In MATLAB, we use the function

$[S, F, T, P]=\operatorname{spectrogram}\left(x\right.$, window, noverlap, $\left.n f f t, f_{S}\right)$

Where $x$ is data, window is a Hamming window of length $n f f$, noverlap is the number of samples that each segment overlaps (the default value is $50 \%$ between segments), $n f f t$ is the FFT length, $f_{s}$ is the sampling frequency in $\mathrm{Hz}$.

See the MATLAB help file for more information.

\section{b) Click removed}

Click signals are usually with high amplitude and will be removed or a very reduced from the data in the time domain after step b). The effort of this operation can be seen in Fig.1(b).

$$
x_{i}^{\prime}=x_{i}^{*} w_{i}
$$

Where $w_{i}$ is calculated using the mean and the standard deviation of the $\mathrm{x}$ in each block of data.

\section{c) Median filter}

The median filter is used to enhance tonal peaks in the spectrogram. For each point of the data, take 50 points on each side of it. And then the median value of the 101 points will be subtracted by the original data. The effort of this operation can be seen in Fig.1(c).

\section{d) Background noise reduction}

Ocean background noise is a long-term stable broadband noise with a certain spectrum structure. In order to extract effective signal, the influence of background noise must be minimized. In this example, besides effective signals, there are also a large number of stable interference signals (around $16 \mathrm{k}$ and $30 \mathrm{k}$ in the example). To remove constant tones from the spectrogram, at each frequency,

$$
x_{t, f}^{\prime}=x_{t, f}-\lambda x_{t-1, f}-\beta x_{t, f-1}
$$

Where $\lambda$ and $\beta$ are variable coefficients. This step can effectively eliminate these noises, and the processing results are shown in Fig.1(d).

\section{e) Gaussian smoothing}

Gaussian smoothing, also known as Gaussian blur, is a linear smoothing filter used for image processing. The principle is to weighted average every pixel of an image, so as to obtain an image with high signal-to-noise. In our method, it adopts third-order Gaussian matrix for processing:

$$
G=\left(\begin{array}{lll}
1 & 2 & 1 \\
2 & 4 & 2 \\
1 & 2 & 1
\end{array}\right) * 1 / 16
$$

\section{f) Threshold control}

The next step is to set the threshold of matrix. The value is $10 \mathrm{~dB}$ in the example. All points below the threshold are set to 0 . See Fig.1(f). 


\section{g) Binaryzation method}

Binarization is also an image processing method. The principle is the gray value of pixel points on the image is set to the highest and the lowest, then the image only presents black and white visual effects. In this method, all elements in the matrix are divided into 0 value and non- 0 value, and the non-0 value is set to 1 .In this way, the target in the image is clear, which provides favorable conditions for subsequent recognition processing. The processing result is shown in Fig.1(g).

\section{h) Graph detection of contours}

The last step is to connect the signal of data matrix into a rectangle region. The signal in the rectangle is considered as a continuous whole. The detection precision can be set by the connection parameters, like c- 8, c-10 (i.e. c- 8 means 8 pixels). At the same time, the former steps are not able to completely eliminate the interference. Therefore, an algorithm needs to be designed to eliminate the remaining interference signals, as shown in Fig.2. The rectangle matrix of the single is determined after the following steps, as shown in Fig. 2:

1. Determine the initial matrix

2. Extension matrix

3. Final matrix determination

4. Remove the interference

5. Determine signal contours

Besides, the following 5 parameters are extracted from each whistle contour: (1) beginning frequency, (2) ending frequency, (3) peak frequency, (4) minimum frequency, (5) duration.

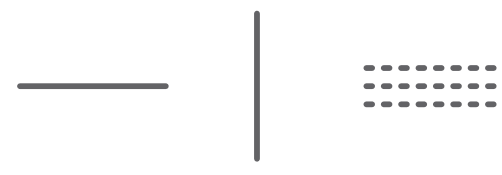

FIG.2. Common types of interference signal.

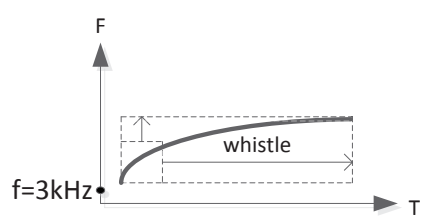

FIG.3. Schematic diagram of extraction principle.

S.

C. Extraction of different whistles

We divide the whistles into 6 types[8][9].

a) Constant signals. A contour where the frequency changes less then $1000 \mathrm{~Hz}$ during the duration.

b) Upsweep. A contour where the frequency is mainly ascending.

c) Downsweep. A contour where the frequency is mainly descending.

d) Convex. A contour includes at least one inflection point, and first ascending then descending.

e) Concave. A contour includes at least one inflection point, and first descending then ascending.

f) Sine. A contour includes at least two inflection points, and first ascending (or descending) then in turn. One Sine signal includes at least three parts edge.

Apply our method to the data of these types, then we get Fig.4.
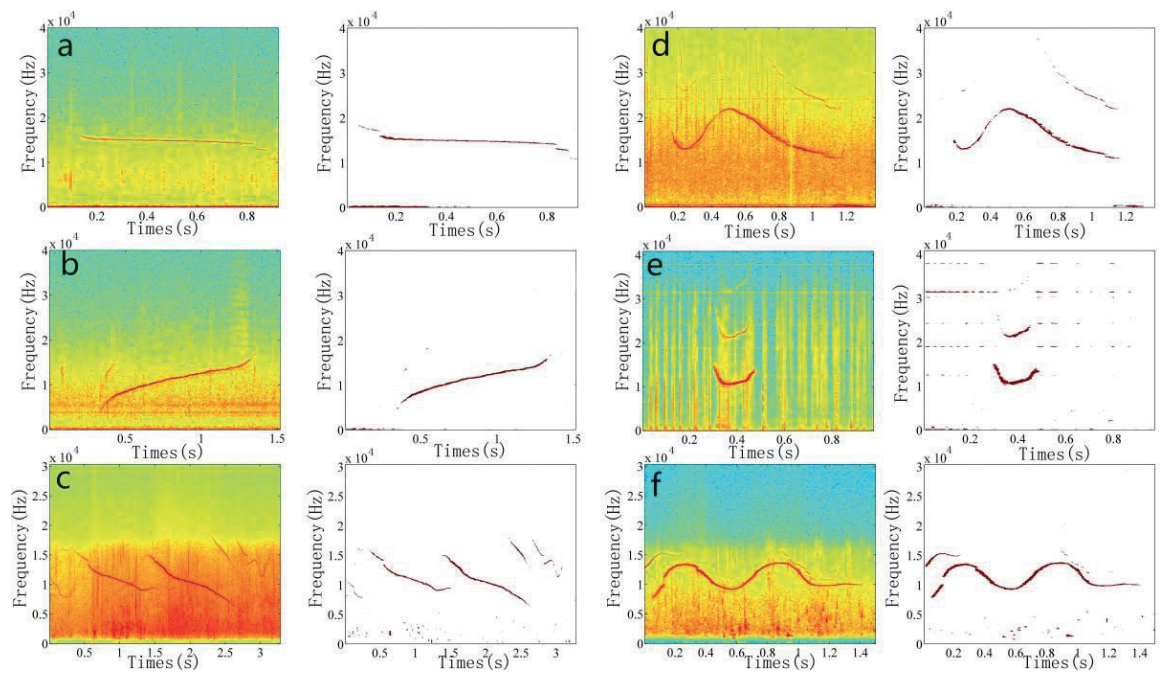
Fig.4.The 6 types of dolphin whistles contours are categorized.

\section{Discussion}

After the invalid signals with too long time and mixed signals removed, 1241 data files were processed in batch, 200 data files were randomly selected for statistical analysis, which contained 376 complete signals. Including 135 the upsweep signal, 57 downsweep signal, 40 sine signal, 31 concave signal, 68 convex signal and 45 constant signal.

After processing, 432 signals were identified, 348 of them were correct. The results show that overall extraction rate is $92.5 \%$ and the effective extraction rate is $80.5 \%$. The reason why the number of identified signal is more than the actual signal, except some error misrecognition, mostly due to the long length of the original signal is broken into two or more segments during the above process.

\section{Conclusion}

In recent years, China has made great efforts to develop Marine investigation, and has accumulated a large number of acoustic data, including mass of valuable Marine mammal acoustic signals. However, there are many difficulties in making effective use of these acoustic data, especially from the ocean background noise. Our algorithm can effectively detect dolphin whistles of various types, even in noise environments. It can also improve future studies on the sound of marine mammals and facilitate long-term passive monitoring on cetacean activity patterns.

\section{Acknowledgments}

We thank Pengsheng Wang and Wanjun Yuan of The Reserve Management Office of Chinese White Dolphins and Amphioxus in Xiamen for their help with sound data acquisition. We also thank the colleagues and members of Third Institute of Oceanography, State Oceanic Administration for their assistance and help during the experiment. Funding for this project was obtained from the Scientific Research Foundation of the Third Institute of Oceanography, SOA (No.2017031 and No.2016026).

\section{References}

[1] Ge Lu Bo He Fu A Г. Dolphins' Hydro locator. Beijing: National
Defense Industry Press. 1984: 10-33.

[2] Lilly, John C., and A. M. Miller. "Sounds Emitted by the Bottlenose Dolphin." Science 133.3465(1961):1689-1693.

[3] Niu, Fu Qiang, et al. "Vocalization and signal characteristic of bottlenose dolphin." Technical Acoustics 30.2(2011):148-152.

[4] Azevedo, Alexandre F., and M. V. Sluys. "Whistles of tucuxi dolphins (Sotalia fluviatilis) in Brazil: Comparisons among populations." Journal of the Acoustical Society of America 117.1(2005):1456-1464.

[5] Gillespie D, et al. "Automatic detection and classification of odontocete whistles. " Journal of the Acoustical Society of America 134.3(2013):2427-37.

[6] Azevedo, Alexandre F., and M. V. Sluys. "Whistles of tucuxi dolphins (Sotalia fluviatilis) in Brazil: Comparisons among populations." Journal of the Acoustical Society of America 117.1(2005):1456-1464.

[7] Lin, T. H., et al. "An automatic detection algorithm for extracting the representative frequency of cetacean tonal sounds. " Journal of the Acoustical Society of America 134.3(2013):2477.

[8] Bazúadurán, Carmen, and W. W. L. Au. "The whistles of Hawaiian spinner dolphins." Journal of the Acoustical Society of America 112.6(2002):3064.

[9] Bazua Duran, and Maria Del Carmen. "The whistles of Hawai'ian spinner dolphins (Stenella longirostris): Description and geographic variations." (2001). 